\title{
Esclerosis tuberosa en el adulto: Hallazgos en tomogra- fía computada multicorte, presentación de un caso clíni- co y revisión de literatura
}

\author{
Drs. Yessenia Orellana $A^{(1)}$, Eugenio Valdés $M^{(2)}$, Aníbal Alé $C^{(2)}$, Cristián Varela $U^{(3)}$.
}

1. Residente de Radiología. Departamento de Radiología, Clínica Dávila, Santiago - Chile.

2. Interno de Medicina. Facultad de Medicina, Universidad de los Andes, Santiago - Chile.

3. Médico Radiólogo. Facultad de Medicina, Universidad de los Andes, Santiago - Chile.

Tuberous sclerosis in adults: Multi slice computed tomography findings. Case report and literature review

Abstract: Tuberous sclerosis, also known as tuberous sclerosis complex, is an autosomal dominant, multisystemic, neurocutaneous disease characterized by a wide range of hamartomatous lesions involving the skin, brain, kidneys, heart, etc. In most cases, it is diagnosed in childhood. Diagnosis in adult patients is usually made by renal failure and lung or dermatologic alterations. We report the case of a 57-year-old female patient with mental retardation and flank pain. A literature review on imaging manifestations of this rare disease is also provided. Keyword: Hamartoma, Neurocutaneous syndrome, Tuberous sclerosis.

Resumen: La esclerosis tuberosa también conocida como complejo esclerosis tuberosa es una enfermedad autosómica dominante, multisistémica, neurocutánea caracterizada por un amplio espectro de lesiones hamartomatosas que afectan la piel, cerebro, riñones, corazón, etc. La mayoría de las veces es diagnosticada en la niñez. El diagnóstico en pacientes adultos se realiza generalmente por falla renal y alteraciones pulmonares 0 dermatológicas. Presentamos el caso de una paciente de 57 años con discapacidad mental y dolor en ambos flancos, lo que acompañamos de una revisión de la literatura respecto de las manifestaciones imagenológicas de esta rara enfermedad.

Palabras clave: Esclerosis tuberosa, Hamartomas, Síndrome neurocutáneo.

Orellana Y y cols. Esclerosis tuberosa en el adulto: Hallazgos en tomografía computada multicorte, presentación de un caso clínico y revisión de literatura. Rev Chil Radiol 2011; 17 (3): 109-112.

Correspondencia: Dra. Yessenia Orellana Araya / yorellana20@yahoo.com

Trabajo recibido el 27 de julio de 2011, aceptado para publicación el 11 de agosto de 2011.

\section{Introducción}

La esclerosis tuberosa (TS) es una enfermedad neurocutánea autosómica dominante, dentro del grupo de desórdenes conocidos como facomatosis. Se caracteriza por la presencia de tumores hamartomatosos benignos en distintos órganos como consecuencia de la mutación de genes específicos que codifican dos proteínas, ambas supresoras de tumores ${ }^{(1-8)}$. La mayoría de las veces es diagnosticada en la niñez, presentándose con autismo, epilepsia y falla cardíaca. El diagnóstico en pacientes adultos se realiza generalmente por falla renal y alteraciones pulmonares o dermatológicas ${ }^{(3)}$.

\section{Caso Clínico}

Paciente sexo femenino de 57 años con antecedentes de institucionalización por discapacidad mental, en estudio por deterioro progresivo de la función renal. Al examen físico se objetiva aumento de volumen abdominal, asociado a dolor en ambos flancos, motivo por el cual se realiza tomografía computada de abdomen. Este estudio muestra voluminosas masas renales bilaterales heterogéneas con predominio de componentes con densidad grasa, que reemplazan al parénquima renal, compatibles con múltiples angiomiolipomas (Figura $1 \mathrm{a}, \mathrm{b}$ ). En el hígado se identifican múltiples nódulos y masas 
sólidas, algunas de densidad grasa y otras con focos de tejido de densidad grasa sugerentes de lipomas y/o angiomiolipomas (Figura 2).

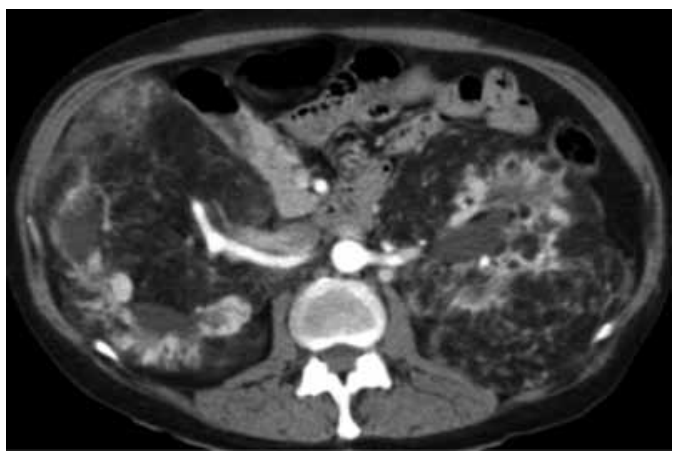

Figura 1. a. Corte axial de TC de abdomen en fase arterial, donde se observan ambos riñones significativamente aumentados de volumen, con su parénquima prácticamente reemplazado por múltiples lesiones heterogéneas, cuya densidad predominante corresponde a tejido adiposo, compatibles con angiomiolipomas.

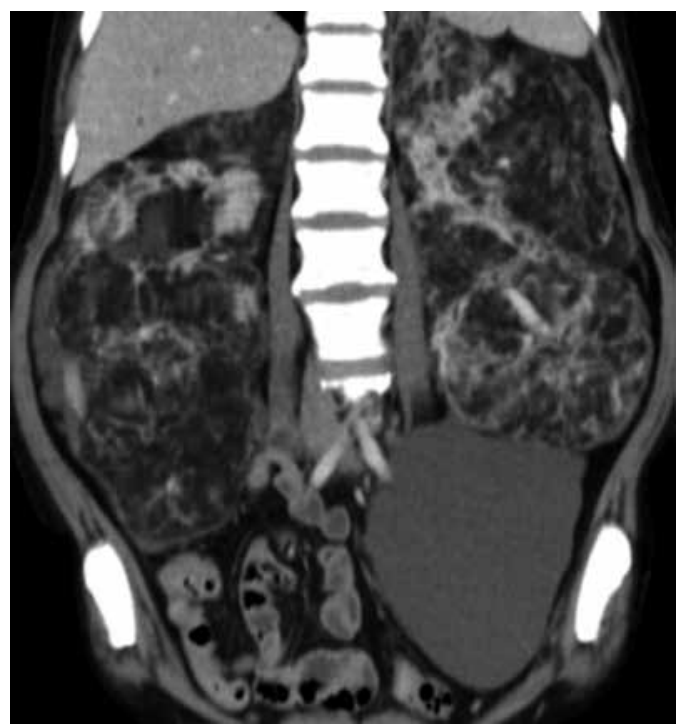

Figura 1. b. Corte coronal de TC en fase portal, donde es patente el gran tamaño alcanzado por los riñones secundario a los innumerables angiomiolipomas. Los riñones ocupan ambos flancos y distienden la pared abdominal. En el riñón izquierdo existe un gran quiste cortical.

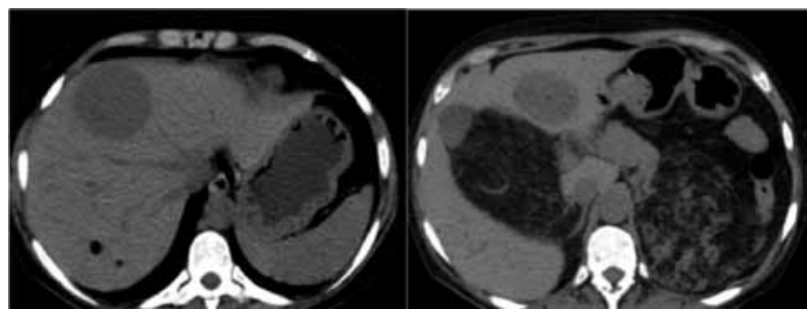

Figura 2. Cortes axiales de TC de abdomen sin contraste en donde se identifican múltiples nódulos y masas sólidas, algunas de densidad grasa y otras con focos de tejido de densidad grasa sugerentes de lipomas y/o angiomiolipomas.
Se complementa con TC de tórax en la que se demuestra una enfermedad quística pulmonar caracterizada por múltiples quistes bilaterales de pared delgada, de distribución simétrica en el parénquima, compatible con patrón tipo linfangioleiomiomatosis (Figura $3 \mathrm{a}, \mathrm{b}$ ).

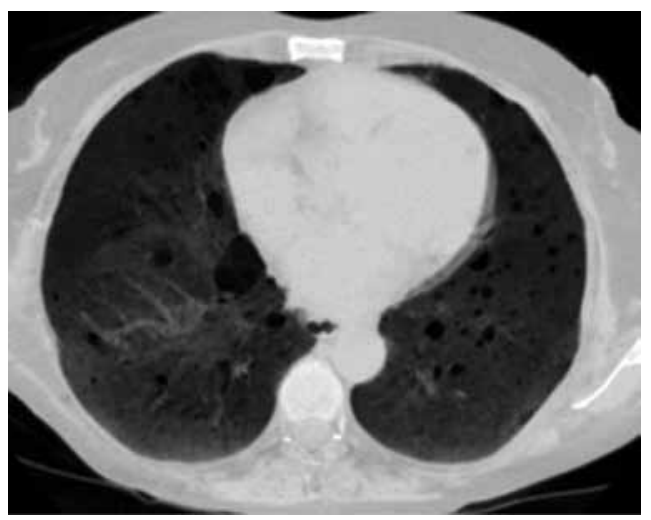

Figura 3. a. Reconstrucción MINIP axial de tórax sin contraste para resaltar múltiples lesiones quísticas de pared delgada y de distribución simétrica compatibles con LAM.

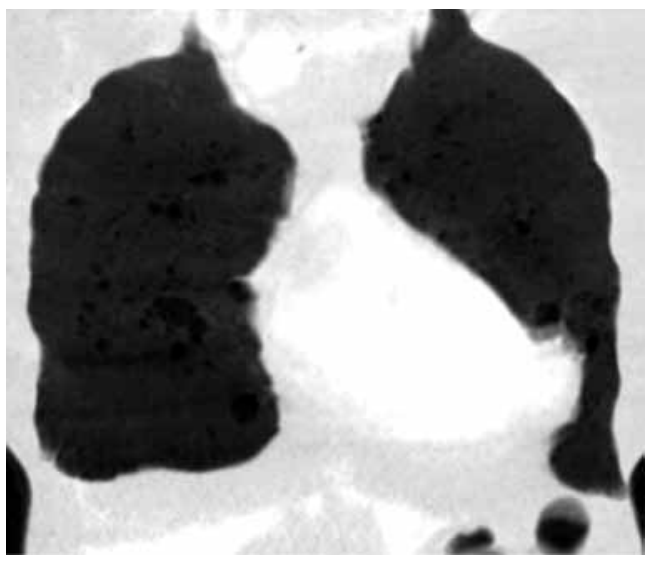

Figura 3. b. Reconstrucción MINIP coronal de tórax sin contraste, demostrando nuevamente las múltiples lesiones quísticas.

El diagnóstico de esclerosis tuberosa en nuestra paciente se confirmó luego de demostrar la presencia de hamartomas subependimarios calcificados en una TC de cerebro (Figura 4).

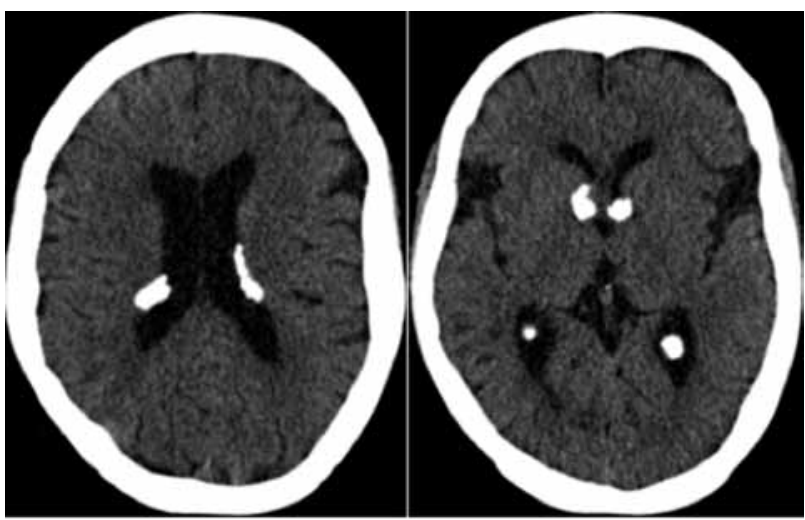

Figura 4. Corte axial de TC de cerebro sin contraste en el que se observan hamartomas calcificados subependimarios. 


\section{Discusión}

La esclerosis tuberosa, también conocida como complejo esclerosis tuberosa o enfermedad de Bourneville-Pringle, es una patología neurocutánea ${ }^{(1-8)}$ caracterizada por la presencia de tumores benignos en distintos órganos como consecuencia de la mutación de dos genes: el TSC 1 que codifica la hamartina y el gen TSC 2 que codifica la tuberina, ambas con función supresora de tumores hamartomatosos ${ }^{(1-5)}$.

La TS se desarrolla principalmente en la infancia y generalmente es diagnosticada antes de los 10 años, 5 siendo la incidencia de 1 cada 10.000 nacimientos $^{(1-6)}$ y alcanza una expectativa de vida de 35 años $^{(2)}$.

Fue descrita por primera vez en 1862 por von Recklinghausen ${ }^{(1,4)}$, pero los primeros criterios diagnósticos fueron establecidos por Voght en 1908 quien estableció la tríada: epilepsia refractaria, discapacidad mental y adenomas sebáceos ${ }^{(1-6)}$.

El diagnóstico actual se basa en los criterios creados en el "Consenso de la Esclerosis Tuberosa" del año $1998^{(1)}$. Los criterios se separan en mayores y menores ${ }^{(1-4)}$ y se basan en la presencia de alteraciones o tumores en distintos órganos (Tabla I) ${ }^{(1,2)}$.
El compromiso cutáneo está dado por máculas hipopigmentadas (90\% de los pacientes), adenoma sebáceo o angiofibroma facial (75\%), que aparecen durante la adolescencia como pequeñas pápulas rojas en el área malar y fibromas periungueales $(20 \%)^{(1-5)}$.

El compromiso neurológico comprende las tuberosidades corticales (90-95\% de los pacientes) que consisten en la pérdida de la estructura normal de las 6 capas de la corteza cerebral(1,2,4). Esta lesión se ha relacionado en un $85 \%^{(1)}$ de los casos con el desarrollo de otras manifestaciones neurológicas como la epilepsia refractaria a tratamiento y la discapacidad mental ${ }^{(1,2,4)}$. Los nódulos subependimales se presentan en la mayoría de los pacientes con TS $(90-95 \%)^{(1)}$, son hamartomas en el tejido subependimal de los ventrículos laterales que con frecuencia se calcifican ${ }^{(1,2,5)}$. Los astrocitomas subependimales de células gigantes (SGAC) tienen una prevalencia de $10-15 \%$ y se ubican con mayor frecuencia en el agujero de Monro, 2,5 son de mayor tamaño que los nódulos subependimales (> $1 \mathrm{~cm})^{(1,2)}$, y pueden producir hidrocefalia obstructiva ${ }^{(2)}$. Por último, las anormalidades de la sustancia blanca incluyen: lesiones quísticas (15-44\%), cercanas a los

Tabla I. Criterios diagnósticos de esclerosis tuberosa.

\section{Criterios mayores}

1 Angiofibromas faciales o placa frontal

2 Fibromas ungueales o periungueales no traumáticos

3 Manchas hipomelanóticas (tres o más)

4 Placas chagrin

5 Hamartomas retinianos nodulares múltiples

6 Tuberosidades corticales

7 Nódulos subependimarios

8 Astrocitoma subependimario de células gigantes

9 Rabdomioma cardíaco, único o múltiple

10 Linfangiomiomatosis pulmonar*

11 Angiomiolipoma renal*

\section{Criterios menores}

Pits múltiples en esmalte dentario

Pólipos rectales hamartomatosos (confirmación histológica)

Quistes óseos (confirmación radiográfica)

Tractos migratorios en la sustancia blanca cerebral $^{* *}$

Fibromas gingivales

Hamartoma no renal (confirmación histológica)

Placa acrómica en retina

Lesiones cutáneas en confeti

Quistes renales múltiples (confirmación histológica)

*Cuando estos dos criterios están presentes se requiere la presencia de otros factores de complejo de esclerosis tuberosa (CTS) para hacer un diagnóstico definitivo.

${ }^{\star *}$ Cuando existe displasia cortical cerebral y tractos migratorios cerebrales en sustancia blanca de forma conjunta deberán contabilizarse como un solo criterio más que como dos criterios separados de CTS.

Diagnóstico definitivo de CTS: dos criterios mayores o un criterio mayor y dos menores. Diagnóstico probable: un criterio mayor y uno menor. Diagnóstico posible: un criterio mayor o más de dos menores. 
ventrículos laterales en la profundidad de ésta y las bandas radiales (15-27\%) ubicadas principalmente en ambos lóbulos frontales ${ }^{(2)}$.

El rabdomioma (50-65\%) es la principal lesión encontrada en el corazón. Es un tumor benigno de las células de músculo liso estriado caracterizado por la presencia de "células araña", llamadas así por sus extensiones citoplasmáticas. Se ubica preferentemente en el septo ventricular y regresa espontáneamente en el $70 \%$ de los pacientes durante los 4 primeros años de vida, por lo que suele ser asintomático. El hallazgo ecográfico incidental de esta lesión determina una probabilidad de un $40-80 \%$ de presentar $\mathrm{TS}^{(1,2)}$.

La linfangioleiomiomatosis (LAM) (26-39\%) es una enfermedad pulmonar quística rara que afecta casi exclusivamente a mujeres jóvenes. Las lesiones características son quistes redondos de tamaño variable y de pared delgada, con distribución simétrica y uniforme ${ }^{(2)}$.

El compromiso renal $(80 \%)^{(1-7)}$, incluye: el angiomiolipoma (AML), quistes renales y carcinoma de células renales que se produce raramente ${ }^{(1,2,3)}$. El AML $(55-75 \%)$ es un tumor benigno cortical, bilateral, compuesto por vasos sanguíneos, células inmaduras de músculo liso y grasa, que se manifiesta en pacientes jóvenes ${ }^{(1,2,3)}$. El número de lesiones renales (AML y quistes) aumenta con la edad, tal como lo observado en nuestra paciente quien se encuentra en una edad avanzada para el promedio de los pacientes con esta enfermedad y posee innumerables tumores ${ }^{(1)}$. En aproximadamente el $87 \%$ de los casos se manifiesta como dolor abdominal, nauseas, vómitos, anemia, hematuria e hipertensión ${ }^{(1,5)}$. Las complicaciones renales son la principal causa de muerte, ya sea por sangrado secundario a ruptura de aneurismas, lo que se encuentra en directa relación al tamaño del
AML, en especial cuando es mayor a $4 \mathrm{~cm}^{(1,4,7)}$ o por insuficiencia renal ${ }^{(5)}$.

Se pueden encontrar también distintos tipos de lesiones en el hígado como lipomas, hamartomas, fibromas y AML (23-45\%) los que se comportan de manera similar que en el riñón, pero con un crecimiento más lento y sin riesgo de muerte por sangrado(1).

En el caso de la paciente de 57 años que acudió a control imagenológico en nuestro centro, presentaba discapacidad mental, angiofibromas faciales, máculas hipopigmentadas, nódulos subependimales, LAM, AML renales bilaterales de gran tamaño, con falla renal secundaria y lesiones hepáticas. Esto confirma el diagnóstico de esclerosis tuberosa, ya que cumple con 4 criterios diagnósticos mayores y 1 menor.

\section{Bibliografía}

1. Borkowska J, Schwartz R, et al. Tuberous sclerosis complex: tumors and tumorigenesis. International Journal of Dermatology 2011; 50: 13-20.

2. Umeoka S, Koyama T, et al. Pictorial Review of Tuberous Sclerosis in Various Organs. RadioGraphics 2008; 10.114/rg.e32 (artículo exclusivo online).

2. Alp A, Sezer SD, Tanrisev M. Tuberous sclerosis diagnosed in adult age. Pak J Med Sci 2011; 27(1): 208-211.

3. Azizul H, Llaila Shamima $S$, et al. Tuberous sclerosis complex in a family. J medicine 2010; 11: 94-98.

4. Ogunrin A, Olubunmi. Misdiagnosis of tuberous sclerosis in a Nigerian girl: A case report and review of literature. Annals of African Medicine 2010; 9(2): 95-101.

5. Sun $\mathrm{H}$, Ethan P. Tuberous Sclerosis Complex. Medicine \& Health/Rhode Island 2010; 93(6): 189-190.

6. Abdelhak K, Younes A, et al. Concurrent Bilateral Renal Angiomyolipoma and Renal Cell Carcinoma in a Patient With Tuberous Sclerosis Complex. Rev Urol. 2009; 11(4): 216-218.

7. Thampi John Nirmal. Tuberous Sclerosis Complex. Urol J 2010; 7(4): 224. 